\title{
Diagnostic application of genotypic identification of mycobacteria
}

\author{
Wing-Cheong Yam, ${ }^{1}$ Kwok-Yung Yuen, ${ }^{1}$ Sin-Yee Kam, ${ }^{1}$ Lap-San Yiu, ${ }^{2}$ \\ Kin-Sang Chan, ${ }^{2}$ Chi-Chiu Leung, ${ }^{3}$ Cheuk-Ming Tam, ${ }^{3}$ Po-On Ho, ${ }^{1}$ \\ Wing-Wai Yew, ${ }^{4}$ Wing-Hong Seto ${ }^{1}$ and Pak-Leung $\mathrm{Ho}^{1}$
}

\section{Correspondence \\ Pak-Leung Ho \\ plho@hkucc.hku.hk}

Received 16 August 2005 Accepted 12 January 2006

\begin{abstract}
Centre of Infection and Department of Microbiology, Queen Mary Hospital, The University of Hong Kong ${ }^{1}$, Pulmonary and Palliative Care and Medical Laboratory, Haven of Hope Hospital ${ }^{2}$, Tuberculosis and Chest Service, Department of Health ${ }^{3}$, and Tuberculosis and Chest Unit, Grantham Hospital ${ }^{4}$, Hong Kong Special Administrative Region, China
\end{abstract}

\section{INTRODUCTION}

Identification of mycobacteria to the species level is important for epidemiological, public health and therapeutic reasons. Traditionally, mycobacteria are identified by phenotypic traits, such as morphological features, growth rates, preferred growth temperature, pigmentation and biochemical profiles. These methods are well established and standardized. However, testing is laborious, difficult and time-consuming, and results are often not reported in a timely manner to guide clinical decisions. Furthermore, misidentification may occur, because different species may have indistinguishable morphological and biochemical profiles. This is especially true for the newly described species (Brown-Elliott et al., 2002), for which the characteristic phenotypic patterns are either absent or unknown. Accordingly, it is necessary for the clinical laboratory to

Abbreviations: MAC, Mycobacterium avium complex; MTB, Mycobacterium tuberculosis. consider alternative strategies to address these limitations of the conventional approach. Mycobacterial fatty acid analysis by GLC represents one such technique, and is commonly applied in regional and reference laboratories. The GLC method is well-established, and systems incorporating computer-assisted analysis of fatty acid identities have been commercially developed for routine application (Larsson et al., 1985; Chou et al., 1998; Torkko et al., 2003). Nonetheless, GLC requires subculture of the primary growth, and 3-5 weeks are often necessary to obtain sufficient cells for analysis (Torkko et al., 2003).

In the last decade, advances in molecular methods have facilitated the rapid and reliable identification of many mycobacterial species by molecular means. Nucleic acid probes, species-specific PCR, reverse hybridization and $16 \mathrm{~S}$ rRNA sequencing have been evaluated for application in clinical laboratories (Cloud et al., 2002; El Amin et al., 2000; Goto et al., 1991; Hall et al., 2003; Kirschner et al., 1993; Lebrun et al., 1992; Patel et al., 1997, 2000; Sarkola et al., 
2004). Due to its ease of use and commercial availability, an acridium ester-labelled probe assay (AccuProbe, GenProbe Inc.) is now incorporated into the workflow of many mycobacterial laboratories (Goto et al., 1991; Lebrun et al., 1992). Specific probes for the identification of Mycobacterium tuberculosis (MTB) complex, Mycobacterium avium complex (MAC), Mycobacterium gordonae and Mycobacterium kansasii have been developed. Probes for the differentiation of members of the MAC into M. avium and Mycobacterium intracellulare are also available. Due to its high analytical sensitivity, species-specific PCR has the added advantage that it may be applied directly to clinical specimens. For the MTB complex and MAC, studies have shown the possibility of nucleic acid detection in smear-negative clinical specimens (Li et al., 1996; Yam et al., 1998; Yuen et al., 1997). The utility of nucleic acid probes and species-specific PCR is, however, limited by the range of mycobacteria which can be identified. Therefore, sequencing of the $16 \mathrm{~S}$ rRNA was developed for identification of mycobacteria. The findings thus far suggest that this technique is rapid, highly discriminative and reliable (Cloud et al., 2002; Hall et al., 2003; Holberg-Petersen et al., 1999; Kirschner et al., 1993; Patel et al., 2000).

In the present study, we report our evaluation of phenotypic methods, GLC and three genotypic tests for identification of mycobacteria, as well as our experience of their integration into a clinical laboratory.

\section{METHODS}

Specimens and isolates. Between July 2001 and May 2004, a total of 1067 Mycobacterium strains were isolated from respiratory and non-pulmonary specimens of 932 patients in Hong Kong, including 342 patients in Queen Mary Hospital, 437 patients in Grantham Hospital, 68 patients in Haven of Hope Hospital and 85 outpatients in chest clinics of the Department of Health. All mycobacteria were identified to species, group or complex level according to growth rate, growth at temperatures of $25,30,35$ and $42{ }^{\circ} \mathrm{C}$, colony morphology, pigmentation, photoreactivity, and a battery of biochemical tests (niacin accumulation, nitrate reduction, semi-quantitative catalase, Tween 80 hydrolysis, tellurite reduction, arylsulfatase and urease production, $\mathrm{NaCl}$ tolerance, and carbohydrate-utilization tests), as described previously (Witebsky et al., 1996; Vincent et al., 2003; Brown-Elliott et al., 2002). The selection of biochemical tests was made according to growth rate, pigment production and colony morphology. Mycobacteria forming visible colonies within 7 days upon subculture were classified as rapid growers (Runyon group IV). Based on production of pigments, the slowly growing organisms were classified into three groups: photochromogen, scotochromogen and nonchromogen, corresponding to Runyon groups I, II and III respectively. The nomenclature of mycobacterial species, complex and group in the latest edition of the Manual of Clinical Microbiology was adopted in the present study (Pfyffer et al., 2003).

GLC analysis of mycobacterial cellular fatty acids. Selected mycobacterial isolates were subcultured onto Middlebrook 7H10 agar supplemented with oleic acid/albumin/dextrose/catalase (OADC) and incubated at $35^{\circ} \mathrm{C}$ for $1-5$ weeks until heavy growth of colonies was observed. A mass of mycobacterial cells sufficient for analysis was saponified, methylated and extracted according to the instructions provided by the manufacturer (MIDI). Whole-cell fatty acid analysis was performed by a Sherlock Microbial Identification SystemAgilent model 6890 gas chromatograph equipped with a flame-ionization detector controlled by Sherlock MIS software (version 4.5). The chromatograms were integrated automatically, and fatty acid identities and percentages were determined using Sherlock patternrecognition software. The identification of the eluted substances was accomplished by comparing the retention times with that of an external calibration standard provided by the manufacturer. The standard provided was a mixture of straight-chain saturated fatty acids from 9 to 20 carbons in length $(9: 0$ to $20: 0)$ and of 5-hydroxy fatty acids. A reference strain, Stenotrophomonas maltophilia (ATCC 13637), was run in parallel as control.

AccuProbe assay. Selected isolates were subjected to AccuProbe assay for MTB complex, MAC, M. gordonae or M. kansasii, depending on the pigmentation and growth rate of the isolates. This assay uses acridium ester-labelled DNA probes and the principle of a hybridization protection assay (Arnold et al., 1989). Testing was performed in accordance with the manufacturer's instructions. After lysis, a $100 \mu \mathrm{l}$ sample was transferred to the corresponding AccuProbe tube. Hybridization results, expressed as relative light units (RLUs), were determined with a Leader 50 luminometer (GenProbe). A positive reaction was a result greater than the cutoff value of $30000 \mathrm{RLU}$, with a repeat range of 20000 to $29999 \mathrm{RLU}$.

DNA extraction for in-house PCR and partial 16S rRNA gene sequencing. Mycobacterial DNA extraction was modified from our previously published protocol (Woo et al., 2003). Briefly, inocula of mycobacterial colonies on LJ medium were washed with $500 \mu \mathrm{l}$ $0 \cdot 1 \mathrm{M}$ Tris/ $\mathrm{HCl}(\mathrm{pH} 7 \cdot 5)$, and the pellet was suspended in $100 \mu \mathrm{l}$ lysis solution containing $0.1 \% \mathrm{NaOH}$ and $0.025 \%$ SDS. The mixture was incubated at $60{ }^{\circ} \mathrm{C}$ for $45 \mathrm{~min}$, followed by addition of $100 \mu \mathrm{l} 0 \cdot 1 \mathrm{M}$ Tris/ $\mathrm{HCl}(\mathrm{pH} 7 \cdot 5)$. The DNA extract was stored at $-20{ }^{\circ} \mathrm{C}$ before PCR.

In-house PCR for MTB complex and MAC. Each PCR reaction contained $10 \mu \mathrm{l}$ DNA extract. A manual one-tube nested PCR for IS6110 in MTB complex was performed as described previously (Chan et al., 1996; Drancourt et al., 2000; Yam et al., 1998; Yuen et al., 1997). PCR for MAC was performed according to the protocol published by $\mathrm{Li}$ et al. (1996), with minor modifications. Of the two forward primers, MAC1 (5'-GGACCTCAAGACGCATGTCTTCTG-3') was derived from positions 138-161 of the 16S rRNA gene of M. avium, and MAC2 (5'-GGACCTTTAGGCGCATGTCTTTAG-3') was derived from positions $128-151$ of the M. intracellulare $16 \mathrm{~S}$ rRNA gene. The design of the reverse primer MACR (5'-GCTCTTTACGCCCAGTAATTCCGG- $3^{\prime}$ ) was based on a sequence which is common to both species. A $100 \mu \mathrm{l}$ reaction mixture consisted of $10 \mathrm{mM}$ Tris/ $\mathrm{HCl}\left(\mathrm{pH} 8.3\right.$ ), $50 \mathrm{mM} \mathrm{KCl}, 2 \mathrm{mM} \mathrm{MgCl}_{2}, 0.2 \mathrm{mM}$ dNTP, $0 \cdot 4 \mu \mathrm{M}$ each primer, and $2 \mathrm{U}$ AmpliTaq Gold polymerase (Perkin Elmer). To activate the Taq polymerase, the mixture was first incubated at $94{ }^{\circ} \mathrm{C}$ for $10 \mathrm{~min}$. The reaction mixture was then subjected to 50 cycles of amplification $\left(94^{\circ} \mathrm{C}\right.$ for $1 \mathrm{~min}, 68^{\circ} \mathrm{C}$ for $1 \mathrm{~min}$ and $72{ }^{\circ} \mathrm{C}$ for $1 \mathrm{~min}$ ), with a final single extension at $72^{\circ} \mathrm{C}$ for $10 \mathrm{~min}$. A $10 \mu \mathrm{l}$ aliquot of PCR product was electrophoresed for $1 \mathrm{~h}$ through a $2 \%$ agarose gel in $1 \times \mathrm{TBE}$, and the target band of 390 bp was visualized under UV illumination.

Partial 16S rRNA gene sequencing. The broad-range primer 285 (5'-GAGAGTTTGATCCTGGCTCAG-3') and the mycobacteriaspecific primer 264 (5'-TGCACACAGGCCACAAGGGA-3') were used for amplification, corresponding to Escherichia coli 16S rRNA positions 9-30 and 1027-1046, respectively (Kirschner et al., 1993). The size of the amplicon was about $1040 \mathrm{bp}$, depending on species. PCR was carried out in a $50 \mu \mathrm{l}$ reaction mixture containing $5 \mu \mathrm{l}$ DNA template, $10 \mathrm{mM}$ Tris/ $\mathrm{HCl}(\mathrm{pH} 8 \cdot 3), 50 \mathrm{mM} \mathrm{KCl}, 2 \mathrm{mM}$ $\mathrm{MgCl}_{2}, 0 \cdot 2 \mathrm{mM}$ dNTP, $0 \cdot 4 \mu \mathrm{M}$ each primer, and $2 \mathrm{U}$ AmpliTaq Gold polymerase. To activate the Taq polymerase, the mixture was 
first incubated at $94{ }^{\circ} \mathrm{C}$ for $10 \mathrm{~min}$. The reaction mixture was then subjected to 35 cycles of amplification $\left(94^{\circ} \mathrm{C}\right.$ for $1 \mathrm{~min}, 65^{\circ} \mathrm{C}$ for $1 \mathrm{~min}$ and $72{ }^{\circ} \mathrm{C}$ for $2 \mathrm{~min}$ ), with a final single extension at $72{ }^{\circ} \mathrm{C}$ for $10 \mathrm{~min}$. A $5 \mu \mathrm{l}$ aliquot of the PCR product was electrophoresed for $1 \mathrm{~h}$ through a $2 \%$ agarose gel in $1 \times$ TBE. Subsequently, PCR products were purified using the QIAquick PCR Purification kit (Qiagen) to remove the unpolymerized primers and deoxynucleoside triphosphates before cycle sequencing. Nucleotide sequences from both DNA strands were determined using the same forward primer 285 and reverse primer 259 (5'-TTTCACGAACAACGCGACAA-3') corresponding to E. coli $16 \mathrm{~S}$ rRNA position 590-609 (Kirschner et al., 1993). The purified fragment was subjected to cycle sequencing by the ABI PRISM Dye Terminator Cycle Sequencing Ready Reaction kit (version 3.0), at a quarter of the recommended reaction volume, and an ABI 377 Genetic Analyser (Applied Biosystems). For all samples, the sequences of both strands of the amplicons were determined. The generated sequences were assembled and edited using the EDITSEQ version 4.0 program in the DNASTAR software (Lasegene), followed by a FASTA search in Ribosomal Differentiation of Medical Micro-organisms (RIDOM) (http://www.ridom-rdna.de/). A sequence match of $\geqslant 99 \%$ with that of the prototype strain sequence in a repository was used as the criterion for species, group or complex level identification (Drancourt et al., 2000). A sequence was considered adequate if the edited sequence had $1 \%$ or less ambiguity (Drancourt et al., 2000). A reference strain, Mycobacterium smegmatis (ATCC 700084), was run in parallel as control. The person performing the sequence protocol was not informed of the identity of the strains by the conventional methods and GLC.

\section{RESULTS AND DISCUSSION}

\section{Identification by conventional method}

Of the 1167 isolates isolated during the study period, the conventional method identified 1157 to the species, group or complex level. They included 895 MTB complex, 118 MAC, 57 Mycobacterium fortuitum group, 32 Mycobacterium chelonae/Mycobacterium abscessus, $20 \mathrm{M}$. gordonae, $18 \mathrm{M}$. kansasii, 12 Mycobacterium marinum, three Mycobacterium terrae complex, one Mycobacterium asiaticum and one Mycobacterium szulgai. The remaining ten unidentified isolates comprised four rapidly growing mycobacteria, three scotochromogens, two nonchromogens and one photochromogen. The conventional tests that we routinely used in our laboratory were unable to differentiate the species within the
MTB complex, the MAC, the M. terrae complex, the $M$. fortuitum group, and the group containing M. chelonae and M. abscessus.

\section{Comparison of conventional method with Accuprobe assays and in-house PCRs}

Among the 1167 clinical isolates, 365 mycobacteria (209 MTB complex, 118 MAC, 20 M. gordonae and 18 M. kansasii) were tested with the Accuprobe assays and in-house PCRs for MTB complex and MAC. The results showed that the results for the conventional method and the two genotypic tests were in complete concordance (Table 1).

\section{Comparison of conventional method with GLC and $16 S$ rRNA gene sequencing}

Among the 1167 clinical isolates, 142 mycobacteria were tested by both GLC and 16S rRNA gene sequencing. This subset included a random sample of 127 isolates chosen to represent the common mycobacteria (MTB complex, MAC, M. fortuitum group, M. gordonae, M. chelonae/M. abscessus, $M$. kansasii and M. marinum); all five infrequent mycobacteria (M. szulgai, M. asiaticum and M. terrae complex), and all ten unidentified isolates (Table 2). All isolates were subjected to identification by conventional tests, GLC and $16 \mathrm{~S}$ rRNA gene sequencing simultaneously. Of the 142 isolates, GLC and $16 \mathrm{~S}$ rRNA sequencing (at $\geqslant 99 \%$ sequence match) identified $137(96.4 \%)$ and $139(97 \cdot 9 \%)$ isolates, respectively, to species, complex or group level. Table 2 shows that concordant results at species, complex or group level were obtained by GLC and 16S rRNA sequencing for all 132 isolates that could be identified by conventional tests. For the ten isolates that could not be identified in the conventional tests, several problematic and discrepant results were obtained by the three methods. Firstly, the fatty acid profiles by GLC of Mycobacterium interjectum were similar to those of Mycobacterium scrofulaceum. Since the former species is not included in the GLC software that we used, both scotochromogens were identified as M. scrofulaceum by GLC, whereas $16 \mathrm{~S}$ rRNA analysis identified both as $M$. interjectum/Mycobacterium simiae. Secondly, despite the fact

Table 1. Comparison of Accuprobe assay and two in-house PCRs with conventional tests, for 365 clinical isolates of mycobacteria

Abbreviations: +, positive; -, negative; ND, not done.

\begin{tabular}{|c|c|c|c|c|c|c|c|c|c|c|c|c|}
\hline \multirow{2}{*}{$\begin{array}{l}\text { Identification by } \\
\text { conventional tests }\end{array}$} & \multicolumn{4}{|c|}{ In-house PCR } & \multicolumn{8}{|c|}{ Accuprobe assay } \\
\hline & \multicolumn{2}{|c|}{ MTB complex } & \multicolumn{2}{|c|}{ MAC } & \multicolumn{2}{|c|}{ MTB complex } & \multicolumn{2}{|c|}{ MAC } & \multicolumn{2}{|c|}{ M. gordonae } & \multicolumn{2}{|c|}{ M. kansasii } \\
\hline MTB complex & 209 & 0 & 0 & 209 & 209 & 0 & 0 & 97 & $\mathrm{ND}$ & $\mathrm{ND}$ & $\mathrm{ND}$ & ND \\
\hline MAC & 0 & 118 & 118 & 0 & 0 & 52 & 118 & 0 & $\mathrm{ND}$ & $\mathrm{ND}$ & ND & ND \\
\hline
\end{tabular}


Table 2. Comparison of identification by GLC and $16 \mathrm{~S}$ rRNA sequencing with conventional methods, for 142 mycobacterial isolates

The number of isolates is shown in parentheses.

\begin{tabular}{|c|c|c|c|}
\hline \multirow{2}{*}{$\begin{array}{l}\text { Organism(s) identified by } \\
\text { phenotypic methods }\end{array}$} & \multirow{2}{*}{$\begin{array}{l}\text { No. of } \\
\text { isolates }\end{array}$} & \multicolumn{2}{|c|}{ Mycobacterial identification method } \\
\hline & & GLC & 16S rRNA sequencing* \\
\hline MTB complex & 36 & MTB complex (36) & MTB complex (36) \\
\hline MAC & 29 & MAC (29) & M. avium (2), M. intracellulare (27) \\
\hline M. fortuitum group & 19 & M. fortuitum (19) & M. fortuitum (19) \\
\hline M. gordonae & 9 & M. gordonae (9) & M. gordonae (9) \\
\hline M. chelonae/M. abscessus & 19 & M. chelonae complex (19) & M. chelonae/M. abscessus (19) \\
\hline M. kansasii & 10 & M. kansasii (4), M. gastri (6) & M. kansasii/M. gastri (10) \\
\hline M. marinum & 5 & M. marinum (5) & M. marinum/M. ulcerans (5) \\
\hline M. szulgai & 1 & M. szulgai (1) & M. szulgai (1) \\
\hline M. asiaticum & 1 & M. asiaticum (1) & M. asiaticum (1) \\
\hline M. terrae complex $\dagger$ & 3 & Mycobacterium nonchromogenicum (3) & M. terrae (3) \\
\hline \multicolumn{4}{|l|}{ Unidentified } \\
\hline Scotochromogens & 3 & M. scrofulaceum (2), no match (1) & M. interjectum/M. simiae (3) \\
\hline Photochromogens & 1 & No match (1) & Mycobacterium lentiflavum (1) \\
\hline Nonchromogens & 2 & M. terrae complex (2) & M. poriferae-like (2) †† \\
\hline Rapid growers & 4 & M. terrae complex (1), $\dagger$ no match (3) & $\begin{array}{l}\text { Mycobacterium chlorophenolicum (1), } \\
\text { Mycobacterium mucogenicum (1), } \\
\text { Mycobacterium neoaurum (1), } \\
\text { Mycobacterium wolinsky-like (1) }\end{array}$ \\
\hline
\end{tabular}

${ }^{*}$ Result obtained when organisms were identified to species, group or complex level by using $\geqslant 99 \%$ sequence match.

$\dagger$. terrae complex consists of three species: M. terrae, M. nonchromogenicum and Mycobacterium trivale. Members in this complex are slowgrowing nonchromogens. $M$. poriferae is a rapid grower.

¥Match at <99\% level: strain HKM159 (98.1\% match with M. wolinsky), strain HKM160 (95.4\% match with M. poriferae), strain 158 (95.6\% match with $M$. poriferae).

that the two nonchromogens were identified as $M$. terrae complex by GLC, no sequence matches at $\geqslant 99 \%$ were obtained in the $16 \mathrm{~S}$ rRNA sequencing. These two isolates had the closest sequence match with Mycobacterium poriferae (95.4-95.6\% similarities), and in fact may represent a novel species. Thirdly, one rapidly growing mycobacterium was identified as M. terrae complex by GLC. This is likely to be erroneous, as all members of the $M$. terrae complex are slow growing. Of the $118 \mathrm{MAC}$, the $16 \mathrm{~S}$ rRNA sequencing identified 116 as M. intracellulare and two as M. avium.

\section{Comparison of turnaround times}

During the present study, inoculated LJ medium was examined daily in the first week, and weekly thereafter. Upon detection of visible growth, Ziehl-Neelsen staining was performed to confirm mycobacterial growth. Depending on the tests and amount of growth, phenotypic and genotypic tests were conducted either directly or after subculture. During the study period, all the genotypic tests were performed two to three times per week. The turnaround time from detection of visible growth on $\mathrm{LJ}$ medium to mycobacterial identification is shown in Table 3.
To the best of our knowledge, this study is unique in evaluating mycobacterial identification by several methods under routine operational conditions. This is an important issue, because workflow and the frequency of testing have an important influence on the length of time required for mycobacterial identification. Due to manpower and resource constraints, many laboratories, including ours, routinely perform only two or three runs of genotypic tests per week. Upon the special request of a clinician, individual testing will be conducted on the same day as growth detection. Therefore, in considering the turnaround time for mycobacterial identification, it is important to take into account the lag time imposed by operational limitations. Therefore, although the AccuProbe hybridization and PCR assays could both be completed within one day, the actual turnaround time was between 2 and 3 days.

This study found 16S rRNA sequencing to be an excellent tool for mycobacterial species identification. Overall, sequence-based identification provided more rapid results and fewer unidentified organisms than conventional methods, corroborating the findings of a recent study (Hall et al., 2003). Furthermore, our findings showed that GLC was largely unhelpful for resolving the ten isolates 
Table 3. Comparison of the actual turnaround time for mycobacterial identification by five methods

\begin{tabular}{|lccccc|}
\hline Mycobacterium & \multicolumn{4}{c|}{ Turnaround time (days), mean \pm SD $^{*}$} \\
\cline { 2 - 6 } & Conventional method $\dagger$ & GLC $\ddagger$ & Accuprobe $\ddagger$ & In-house PCR $\ddagger$ & 16S rRNA sequencing $\ddagger$ \\
\hline MTB complex & $16 \pm 7$ & $23 \pm 4$ & $3 \pm 1$ & $2 \pm 1$ & $4 \pm 2$ \\
Non-tuberculous mycobacteria & $23 \pm 10$ & $29 \pm 5$ & $3 \pm 2$ & $2 \pm 1$ & $5 \pm 2$ \\
MAC & $22 \pm 9$ & $14 \pm 5$ & $3 \pm 2 \$$ & - & $4 \pm 2$ \\
Other slow growers & $7 \pm 2$ & $6 \pm 3$ & $2 \pm 1$ & - & $3 \pm 2$ \\
Rapid growers & & & & \\
\end{tabular}

${ }^{\star}$ Time required for completion of tests for the bacterial identification of an isolated mycobacterium. This did not include the time employed in transport and in culture isolation from clinical specimens.

$\dagger$ For all 1167 mycobacterial isolates.

¥For the isolates tested as detailed in Tables 1 and 2 .

§For M. kansasii and M. gordonae only.

unidentified by conventional methods. Unlike GLC, which requires heavy growth of mycobacteria in standard medium, sequencing of PCR-amplified 16S rRNA products can be performed with less than a single colony. Thus, colonies on LJ medium can be tested directly without subculture if growth is pure. With this direct approach, however, one should note that mixed growth could lead to ambiguous $16 \mathrm{~S}$ rRNA data (Drancourt et al., 2000).

16S rRNA sequencing-based identification has limitations. Firstly, it could not always distinguish between closely related species. The following examples were found in our evaluation: M. chelonae and M. abscessus; $M$. kansasii and Mycobacterium gastri; M. marinum and Mycobacterium ulcerans; and M. interjectum and M. simiae. Secondly, $16 \mathrm{~S}$ rRNA sequencing is unable to discriminate members of the MTB complex: M. tuberculosis sensu stricto, Mycobacterium bovis, Mycobacterium africanum, Mycobacterium microti and Mycobacterium canettii. For differentiation of the above closely related species, or members of the MTB complex, additional biochemical or other tests are required. For example, $M$. chelonae can be distinguished from M. abscessus by its ability to utilize citrate, by susceptibility to tobramycin (MIC of $\leqslant 4 \mu \mathrm{g} \mathrm{ml}^{-1}$ ), or by the RFLP pattern of the $h s p$ gene (Yakrus et al., 2001). M. kansasii and M. marinum can be readily distinguished from the other two related species by their pigmentation. Unfortunately, no single phenotypic or genotypic test is able to readily distinguish all members within the MTB complex. In most diagnostic laboratories, the great majority of isolates are likely to be $M$. tuberculosis sensu stricto. Therefore, it has been suggested that specific tests, such as $o x y R / p n c A$ allele-specific PCR, may be limited to strains with atypical features, such as not yielding rough, cream, cauliflower-like colonies (Vincent et al., 2003). Recently, the GenoType MTBC assay (Hain Lifescience) has also been reported to be useful for differentiation of subtypes within the MTB complex (Richter et al., 2004). Since the assay allows MTB complex identification and subtyping in a single test, it has superseded Accuprobe in some clinical laboratories. Secondly, reliable sequence identification depends on the accuracy of the obtained data as well as that of the reference sequence in the database. Therefore, the obtained sequence should contain no more than $1 \%$ ambiguity and should be analysed against a peerreviewed database such as Ribosomal Differentiation of Medical Micro-organisms (RIDOM).

Despite the many advantages of sequence-based identification, the cost of DNA sequencing is still too expensive (US $\$ 80-130$ per specimen) to be used routinely for all samples in diagnostic laboratories (Clarridge, 2004). This has prompted us to explore strategies for the more cost-effective use of $16 \mathrm{~S}$ rRNA sequencing. While almost 100 members of the mycobacteria are now recognized (Pfyffer et al., 2003), a small proportion of them account for the great majority of clinical isolates: MTB complex, MAC, M. fortuitum group, M. chelonae, M. abscessus, M. kansasii and M. marinum. In the present study, MTB complex and MAC provided $95 \%$ $(1013 / 1067)$ of the total isolates. If the cultured isolates were screened by specific in-house PCR (or AccuProbe assays) for the two complexes, a large proportion of isolates could be readily identified and very few isolates would require sequencing (Fig. 1). With this algorithm, subculture and growth for a short period of time may be necessary to ascertain pure growth and also for the designation of the mycobacterium as a rapid or slow grower. Thereafter, the great majority of isolates could be identified and reported within one week. As a cost-reduction measure, our present experience demonstrates that an adequate result could be obtained by using one-quarter reaction volumes for DNA sequencing. We sought to develop the in-house PCR in lieu of AccuProbe assays for identification of MTB complex and MAC as a means of cost containment. Our in-house PCR for MTB complex targets IS6110 as a highly specific marker for identification purposes. This marker is present in virtually all members of the MTB complex (Vincent et al., 2003). The specificity and sensitivity of this in-house PCR have been extensively evaluated by our group (Yuen et al., 1997; Yam et al., 1998, 2004; Chan et al., 1996). However, it should be noted that some populations have a small number of 


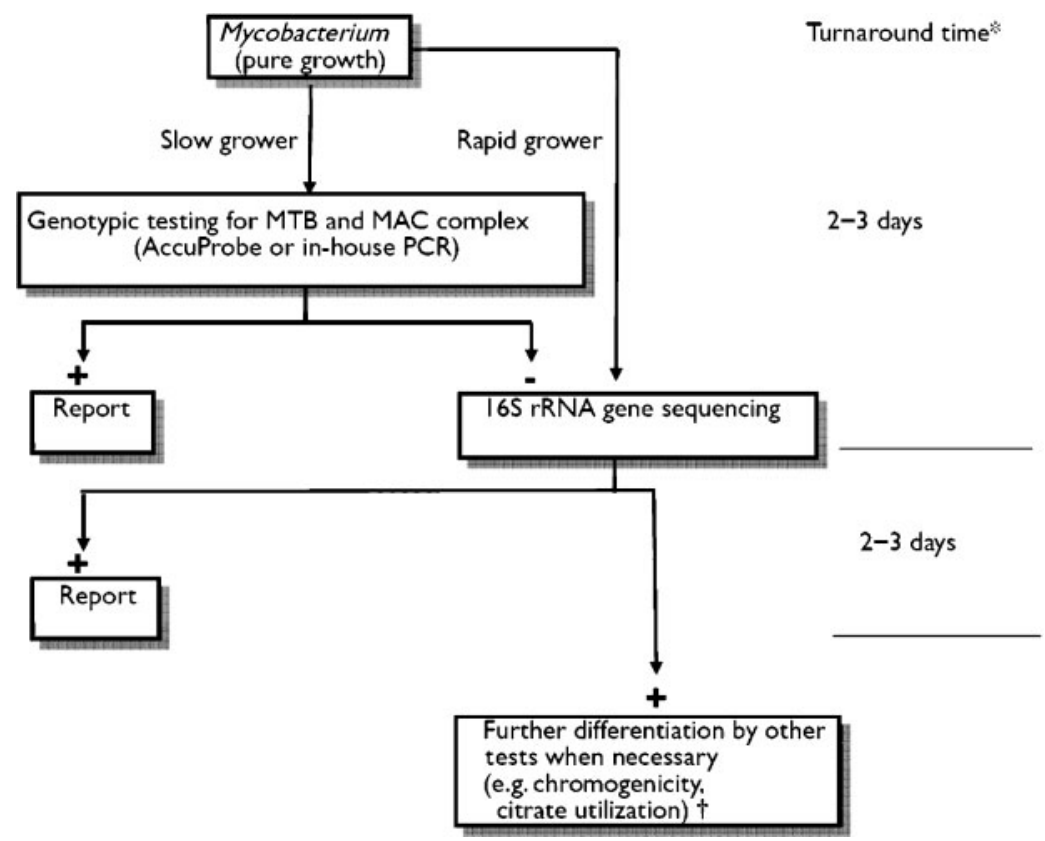

Fig. 1. Algorithm for rapid species identification of Mycobacterium by an integrated approach. *Three runs per week. †ln this study, further differentiation was required to differentiate $M$. chelonae and $M$. abscessus, M. kansasii and M. gastri, and M. marinum and M. ulcerans. +, Positive; -, negative.

IS6110-negative strains (Park et al., 2000). Under the proposed scheme, these IS6110-negative strains would be identified at the $16 \mathrm{~S}$ rRNA sequencing stage. Likewise, the specificities of the primers MAC1, MAC2 and MACR have previously been studied by Li et al. (1996). Depending on the local requirement, both species-specific PCR and AccuProbe assays can be adapted for identification to MAC or species level. The hands-on time for both AccuProbe and in-house PCR are similar (2-3 h) and both can be efficiently completed on the same day. Under operational conditions, this study found that both methods were also equivalent in terms of accuracy and actual turnaround time. With respect to reagents and supplies, the cost of the in-house PCR (approx. US \$5 per test) is lower than that of the AccuProbe test (approx. US \$35 per test). With this integrated scheme (Fig. 1), the majority of the phenotypic tests could be abandoned and the resources channeled into genotypic testing. The main purpose of the remaining phenotypic tests (e.g. growth rate, chromogenicity and citrate utilization) would be to resolve closely related species or members of a complex or group with highly similar 16S rRNA sequences. A difficulty with the AccuProbe test is that a decision needs to be made about which probe to use, according to the morphology of the organism, the time to positive growth, and clinical factors. For sequence-based identification, our experience approximates the earlier estimation of Clarridge (2004) that for three runs of 20 samples per week, the total labour time is about 40-50 h. In our laboratory, we estimate that there would be no overall increase in the laboratory budget, while manpower would be saved by approximately $15 \%$.

We used an in-house protocol for the sequence-based identification. This could limit applicability in other laboratories because of the technical demands of manual DNA extraction, PCR, cycle sequencing and output analysis. The turnaround time for the testing and analysis was about 2 days, which is comparable to that of the commercial MicroSeq 500 system (Patel et al., 2000). Understandably, the required expertise and resources for molecular testing in the present scheme may be lacking in some developing countries. Our scheme is also limited in that the similarity match was relatively low for a small number of isolates. However, this problem is common to all identification based on 16S rRNA sequencing, and has also been reported by other investigators (Cloud et al., 2002; Hall et al., 2003; Patel et al., 2000). The question arises of when to consider a sequence as novel and when it should be considered as closely related to that of an existing species. At present, there are no accepted guidelines available to establish cutoffs for computer-assisted analysis of sequence similarity for $16 \mathrm{~S}$ rRNA-based bacterial identification. Difference rates of $\leqslant 0 \cdot 5$, $\leqslant 1$ and $\leqslant 3 \%$ have been recommended for delineation at the species level (Clarridge, 2004). Since bacterial genera do not evolve at the same speed, it may be necessary to use different cutoff values for different bacterial genera. We used $a \leqslant 1 \%$ difference as a suitable cutoff, as suggested by Drancourt et al. (2000). In terms of commercially available options, assays have been developed that can rapidly identify a broad range of mycobacterial species by amplification of culture material and subsequent reverse hybridization onto strips (Padilla et al., 2004).

It might be argued that for patients with typical presentation, the clinical benefit of speeding up identification of MTB complex by 2 weeks is small, because these patients are already on empirical therapy. However, tuberculosis is well known to have atypical presentations. The management of this patient subset and of those with extra-pulmonary nontuberculous mycobacteria (NTM) infections is likely to 
benefit from the shortened turnaround time for identification of culture material. In terms of practical utility, it is important that genotypic testing be integrated with rapid culture. In many developed countries, automated liquidbased systems are increasingly used for routine mycobacterial culture and sensitivity testing. These systems provide faster speed and greater sensitivity for primary isolation of mycobacteria from clinical specimens. A further advantage is that culture materials are available on a daily basis for genotypic testing; thus, the integration of automated liquid-based systems with genotypic testing will further add to the rapidity of the total laboratory investigation. In our laboratory, the BACTEC Myco/F Lytic system (Becton Dickinson) has also been available since the mid-1990s. Due to budgetary constraints, we have not been able to replace all LJ culture by this liquid-based system. Instead, its use is prioritized to immunocompromised patients, persons with risk factors for drug-resistant tuberculosis, and upon request by the attending clinician.

In conclusion, $16 \mathrm{~S}$ rRNA sequencing offers a rapid and reliable means for mycobacterial identification. Our findings provide a model for how this technique can be integrated with species-specific PCR tests, AccuProbe hybridization assays and conventional tests in diagnostic laboratories in a cost-conscious manner. Interpretation of the sequences without a good match in the database remains problematic. As 16S rRNA sequences become more widely used in diagnostic laboratories, guidelines for and standardization of the delineation of cutoffs for sequence interpretation are required.

\section{ACKNOWLEDGEMENTS}

This work is supported by research grants from the Research Fund for the Control of Infectious Diseases of the Health, Welfare and Food Bureau of the Hong Kong Special Administrative Region Government and from the University Development Fund Project-Research Centre of Emerging Infection Diseases of the University of Hong Kong.

\section{REFERENCES}

Arnold, L. J., Jr, Hammond, P. W., Wiese, W. A. \& Nelson, N. C. (1989). Assay formats involving acridinium-ester-labeled DNA probes. Clin Chem 35, 1588-1594.

Brown-Elliott, B. A. \& Wallace, R. J., Jr (2002). Clinical and taxonomic status of pathogenic nonpigmented or late-pigmenting rapidly growing mycobacteria. Clin Microbiol Rev 15, 716-746.

Brown-Elliott, B. A., Griffith, D. E. \& Wallace, R. J., Jr (2002). Newly described or emerging human species of nontuberculous mycobacteria. Infect Dis Clin North Am 16, 187-220.

Chan, C. M., Yuen, K. Y., Chan, K. S., Yam, W. C., Yim, K. H., Ng, W. F. \& Ng, M. H. (1996). Single-tube nested PCR in the diagnosis of tuberculosis. J Clin Pathol 49, 290-294.

Chou, S., Chedore, P. \& Kasatiya, S. (1998). Use of gas chromatographic fatty acid and mycolic acid cleavage product determination to differentiate among Mycobacterium genavense, Mycobacterium fortuitum, Mycobacterium simiae, and Mycobacterium tuberculosis. J Clin Microbiol 36, 577-579.
Clarridge, J. E., III (2004). Impact of 16S rRNA gene sequence analysis for identification of bacteria on clinical microbiology and infectious diseases. Clin Microbiol Rev 17, 840-862.

Cloud, J. L., Neal, H., Rosenberry, R., Turenne, C. Y., Jama, M., Hillyard, D. R. \& Carroll, K. C. (2002). Identification of Mycobacterium spp. by using a commercial $16 \mathrm{~S}$ ribosomal DNA sequencing kit and additional sequencing libraries. J Clin Microbiol 40, 400-406.

Drancourt, M., Bollet, C., Carlioz, A., Martelin, R., Gayral, J. P. \& Raoult, D. (2000). 16S ribosomal DNA sequence analysis of a large collection of environmental and clinical unidentifiable bacterial isolates. J Clin Microbiol 38, 3623-3630.

El Amin, N. M., Hanson, H. S., Pettersson, B., Petrini, B. \& Von Stedingk, L. V. (2000). Identification of non-tuberculous mycobacteria: $16 \mathrm{~S}$ rRNA gene sequence analysis vs. conventional methods. Scand J Infect Dis 32, 47-50.

Goto, M., Oka, S., Okuzumi, K., Kimura, S. \& Shimada, K. (1991). Evaluation of acridinium-ester-labeled DNA probes for identification of Mycobacterium tuberculosis and Mycobacterium avium-Mycobacterium intracellulare complex in culture. J Clin Microbiol 29, 2473-2476.

Hall, L., Doerr, K. A., Wohlfiel, S. L. \& Roberts, G. D. (2003). Evaluation of the MicroSeq system for identification of mycobacteria by $16 \mathrm{~S}$ ribosomal DNA sequencing and its integration into a routine clinical mycobacteriology laboratory. J Clin Microbiol 41, 1447-1453.

Holberg-Petersen, M., Steinbakk, M., Figenschau, K. J., Jantzen, E., Eng, J. \& Melby, K. K. (1999). Identification of clinical isolates of Mycobacterium spp. by sequence analysis of the $16 \mathrm{~S}$ ribosomal RNA gene. Experience from a clinical laboratory. Acta Pathol Microbiol Immunol Scand 107, 231-239.

Kirschner, P., Springer, B., Vogel, U., Meier, A., Wrede, A., Kiekenbeck, M., Bange, F. C. \& Bottger, E. C. (1993). Genotypic identification of mycobacteria by nucleic acid sequence determination: report of a 2-year experience in a clinical laboratory. J Clin Microbiol 31, 2882-2889.

Larsson, L., Jantzen, E. \& Johnsson, J. (1985). Gas chromatographic fatty acid profiles for characterisation of mycobacteria: an interlaboratory methodological evaluation. Eur J Clin Microbiol 4, 483-487.

Lebrun, L., Espinasse, F., Poveda, J. D. \& Vincent-Levy-Frebault, V. (1992). Evaluation of nonradioactive DNA probes for identification of mycobacteria. J Clin Microbiol 30, 2476-2478.

Li, Z., Bai, G. H., von Reyn, C. F., Marino, P., Brennan, M. J., Gine, N. \& Morris, S. L. (1996). Rapid detection of Mycobacterium avium in stool samples from AIDS patients by immunomagnetic PCR. J Clin Microbiol 34, 1903-1907.

Padilla, E., Gonzalez, V., Manterola, J. M. \& 8 other authors (2004). Comparative evaluation of the new version of the INNO-LiPA Mycobacteria and GenoType Mycobacterium assays for identification of Mycobacterium species from MB/BacT liquid cultures artificially inoculated with mycobacterial strains. J Clin Microbiol 42, 3083-3088.

Park, Y. K., Bai, G. H. \& Kim, S. J. (2000). Restriction fragment length polymorphism analysis of Mycobacterium tuberculosis isolated from countries in the western Pacific region. J Clin Microbiol 38, 191-197.

Patel, S., Yates, M. \& Saunders, N. A. (1997). PCR-enzymelinked immunosorbent assay and partial rRNA gene sequencing: a rational approach to identifying mycobacteria. J Clin Microbiol 35, 2375-2380.

Patel, J. B., Leonard, D. G., Pan, X., Musser, J. M., Berman, R. E. \& Nachamkin, I. (2000). Sequence-based identification of Mycobacterium species using the MicroSeq $50016 \mathrm{~S}$ rDNA bacterial identification system. J Clin Microbiol 38, 246-251.

Pfyffer, G. E., Brown-Elliott, B. A. \& Wallace, R. J. (2003). Mycobacterium: general characteristics, isolation, and staining procedures. 
In Manual of Clinical Microbiology, pp. 532-559. Edited by P. R. Murray, E. J. Baron, J. H. Jorgensen, M. A. Pfaller \& R. H. Yolken. Washington, DC: American Society for Microbiology.

Richter, E., Weizenegger, M., Fahr, A. M. \& Rusch-Gerdes, S. (2004). Usefulness of the GenoType MTBC assay for differentiating species of the Mycobacterium tuberculosis complex in cultures obtained from clinical specimens. J Clin Microbiol 42, 4303-4306.

Sarkola, A., Makinen, J., Marjamaki, M., Marttila, H. J., Viljanen, M. K. \& Soini, H. (2004). Prospective evaluation of the GenoType assay for routine identification of mycobacteria. Eur J Clin Microbiol Infect Dis 23, 642-645.

Torkko, P., Katila, M. L. \& Kontro, M. (2003). Gas-chromatographic lipid profiles in identification of currently known slowly growing environmental mycobacteria. J Med Microbiol 52, 315-323.

Vincent, V., Brown-Elliott, B. A., Jost, K. C., Jr \& Wallace, R. J. (2003). Mycobacterium: phenotypic and genotypic identification. In Manual of Clinical Microbiology, pp. 560-587. Edited by P. R. Murray, E. J. Baron, J. H. Jorgensen, M. A. Pfaller \& R. H. Yolken. Washington, DC: American Society for Microbiology.

Witebsky, F. G. \& Kruczak-Filipov, P. (1996). Identification of mycobacteria by conventional methods. Clin Lab Med 16, 569-601.
Woo, P. C., Ng, K. H., Lau, S. K., Yip, K. T., Fung, A. M., Leung, K. W., Tam, D. M., Que, T. L. \& Yuen, K. Y. (2003). Usefulness of the MicroSeq 500 16S ribosomal DNA-based bacterial identification system for identification of clinically significant bacterial isolates with ambiguous biochemical profiles. J Clin Microbiol 41, 1996-2001.

Yakrus, M. A., Hernandez, S. M., Floyd, M. M., Sikes, D., Butler, W. R. \& Metchock, B. (2001). Comparison of methods for identification of Mycobacterium abscessus and M. chelonae isolates. J Clin Microbiol 39, 4103-4110.

Yam, W. C., Yuen, K. Y. \& Seto, W. H. (1998). Direct detection of Mycobacterium tuberculosis in respiratory specimens using an automated DNA amplification assay and a single tube nested polymerase chain reaction. Clin Chem Lab Med 36, 597-599.

Yam, W. C., Tam, C. M., Leung, C. C. \& 8 other authors (2004). Direct detection of rifampin-resistant Mycobacterium tuberculosis in respiratory specimens by PCR-DNA sequencing. J Clin Microbiol 42, 4438-4443.

Yuen, K. Y., Yam, W. C., Wong, L. P. \& Seto, W. H. (1997). Comparison of two automated DNA amplification systems with a manual one-tube nested PCR assay for diagnosis of pulmonary tuberculosis. J Clin Microbiol 35, 1385-1389. 